\title{
Tratamiento de la rectitis actínica hemorrágica con aplicación tópica de formalina al $4 \%$ *
}

\author{
Drs. ALEJANDRO BARRERA E. ${ }^{1}$, JUAN MANSILLA E. ${ }^{1}$, GUILLERMO BANNURA C. ${ }^{1}$, \\ FELIPE ILLANES F. ${ }^{1}$, JAIME CONTRERAS P. ${ }^{1}$, CLAUDIO ZÚÑIGA T. ${ }^{1}$, \\ KAREN ROJAS R. ${ }^{2}$, BÁRBARA DEL CASTILLO A. ${ }^{3}$, FERNANDO DINAMARCA C. ${ }^{3}$ \\ 1 Servicio y Departamento de Cirugía, Hospital Clínico San Borja Arriarán. Campus Centro, \\ Facultad de Medicina, Universidad de Chile. \\ 2 Interna de Medicina, Universidad de Chile. \\ 3 Alumnos de Medicina, Universidad de Chile. \\ Santiago, Chile.
}

\begin{abstract}
Treatment of hemorrhagic radiation rectitis with topical application of $4 \%$ formaldehyde

Background: Hemorrhagic radiation rectitis occurs in 15\% of patients subjected to pelvic irradiation. One of the treatment alternatives is the topical application of $4 \%$ formaldehyde. Aim: To report the results of the use of topical formaldehyde in radiation rectitis. Material and Methods: Twenty patients aged 36 to 80 years (13 women) with hemorrhagic radiation rectitis were prospectively recruited. Fistula or stenosis was discarded endoscopically. Formaldehyde was applied in the operating room or at the outpatient clinic. Results: Fifteen patients required repeated transfusions. The application was performed in the operating room in five patients. Bleeding stopped in three patients with one application, in 12 patients with two applications and in four, with three applications. In one patient, bleeding did not stop after the first application and had a sigmoid perforation; therefore no further application was attempted. One patient had a severe proctitis after the procedure, which subsided with symptomatic treatment after 15 days. Conclusions: Topical formaldehyde application for hemorrhagic rectitis is effective to stop bleeding but has complications in $10 \%$ of patients.
\end{abstract}

Key words: Radiation rectitis, bleeding, formaldehyde.

\section{Resumen}

Introducción: La rectitis actínica hemorrágica es una complicación que se presenta en el 15\% de los pacientes sometidos a radioterapia por una neoplasia pélvica. Existen distintas alternativas para su tratamiento, entre las que se cuenta la aplicación de formalina al 4\%. Presentamos los resultados del tratamiento de esta complicación en una serie prospectiva no aleatoria. Material y Método: Desde marzo de 2004 a mayo de 2007 se reclutaron en forma prospectiva todos los pacientes tratados por una rectitis actínica hemorrágica

*Recibido el 26 de octubre de 2010 y aceptado para publicación el 29 de diciembre de 2010.

Correspondencia: Dr. Alejandro Barrera E.

Apoquindo 8160 Dpto. 31, Santiago, Chile

abarrera@med.uchile.cl 
en nuestro servicio. En todos los pacientes se descartó una estenosis o fístula por endoscopia flexible. Los pacientes fueron sometidos a aplicación de formalina en pabellón o ambulatoria según la evaluación del tratante. Resultados: La serie corresponde a 20 pacientes, 13 mujeres, con un promedio de edad de 61 años (36-80), 15 requerían transfusiones a repetición. La indicación de radioterapia fue por un cáncer cérvico uterino en 9 casos, de próstata en 7 casos y endometrio en 4 . El promedio de sesiones necesarias para controlar el sangrado fue de 2 (1-3); en 5 pacientes la aplicación se realizó en pabellón. Tres pacientes mejoraron con una aplicación, 12 pacientes con 2 aplicaciones y 4 con 3 procedimientos. Un paciente continúa sangrado después de un procedimiento, pero presenta una perforación de sigmoides en terreno neoplásico y no se intentó una segunda sesión, por lo que se considera un fracaso del procedimiento. Además un paciente presentó una proctitis intensa que cedió con tratamiento sintomático en 15 días, con lo que la morbilidad del procedimiento alcanza a un 10\%. El éxito acumulado es de un 95\% de los casos, con detención del sangrado, sin necesidad de transfusiones. Conclusión: Estimamos que el tratamiento con formalina al 4\% es un procedimiento seguro para el tratamiento de la rectitis actínica hemorrágica, con buenos resultados en la gran mayoría de los pacientes, evitando procedimientos más caros o de mayor morbilidad.

Palabras clave: Rectitis actínica, tratamiento, formalina.

\section{Introducción}

La radioterapia es una forma importante de tratamiento para un número significativo de neoplasias de la pelvis. La ubicación fija del recto y su proximidad a estructuras con el cuerpo y cuello uterino, la próstata y el ano lo hacen especialmente vulnerable al daño secundario a la radioterapia ${ }^{1}$. La incidencia de esta complicación varía entre el 1 y el $20 \%$ dependiendo del momento en que se efectúa el diagnóstico y de su gravedad ${ }^{2}$.

El daño crónico provocado por la radioterapia es un fenómeno progresivo y permanente resultante de la endarteritis obliterante y la consiguiente isquemia de la pared rectal. El síntoma más frecuente es el sangrado rectal, el que puede ser acompañado de erosiones, úlceras, estenosis o fístulas ${ }^{3}$.

Las alternativas de tratamiento son variadas e incluyen terapias medicamentosas y procedimientos mínimamente invasivos con tasas de éxito variables $^{4-6}$. La formalina se utilizó por primera vez en el tratamiento de la cistitis hemorrágica por radiación por Brown ${ }^{7}$ en 1969, y dado sus buenos resultados se comenzó a intentar el sangrado rectal a mediados de los $80^{8}$. Desde entonces distintas series han demostrado su utilidad y seguridad.

El objetivo de esta comunicación es presentar los resultados del tratamiento de la proctitis crónica hemorrágica por radioterapia en una serie prospectiva no aleatoria.

\section{Material y Método}

Desde marzo de 2004 a mayo de 2010 todos los pacientes derivados por rectitis actínica al policlínico de Coloproctología del Hospital Clínico San Borja Arriarán fueron atendidos por el autor. Los pacientes fueron evaluados con una rectoscopia rígi- da y una colonoscopía para confirmar el diagnóstico y para descartar una estenosis rectal o una fístula. Los pacientes fueron clasificados de acuerdo a lo propuesto por Haas ${ }^{1}$, en grados leve, moderado y grave según la cuantía del sangrado y los hallazgos endoscópicos.

Además se efectuó una evaluación por su equipo oncológico tratante para descartar una recidiva local al momento de realizar el tratamiento.

El tratamiento se realizó en pabellón o en sala de examen de acuerdo a la decisión del tratante. Si se efectuó en pabellón el paciente recibió anestesia regional, fue ubicado en posición de litotomía forzada con una abundante lubricación del periné, siendo la formalina al $4 \%$ aplicada con torundas hasta obtener palidez de la mucosa, la que luego fue lavada con solución fisiológica. En el policlínico el paciente fue colocado en posición de Simms con lubricación amplia del periné y un rectoscopio rígido fue colocado inmediatamente proximal a la zona de sangrado. Se instilaron 5 a $10 \mathrm{ml}$ de formalina al 4\%, los que fueron aspirados y lavados con solución fisiológica al lograr la palidez de la mucosa. Se procedió a retirar el rectoscopio 2 a $3 \mathrm{~cm}$ y se repitió el procedimiento en toda la superficie sangrante.

Los pacientes fueron evaluados a las 3 semanas y de persistir el sangrado fue repetido el tratamiento. En los pacientes tratados en policlínico, en que el sangrado persistió después de dos aplicaciones, se realizó una tercera sesión en pabellón bajo anestesia.

Se definió como éxito del tratamiento a la detención del sangrado rectal, la desaparición de la anemia y la falta de necesidad de transfusiones.

Se registró en una base de datos ad hoc los datos epidemiológicos y clínicos de los pacientes, como edad, sexo, indicación de la radioterapia, tiempo de evolución del sangrado, necesidad de transfusiones y respuesta a la terapia. 


\section{Resultados}

La serie corresponde a 20 pacientes, 13 mujeres, con un promedio de edad de 61 años (36-80). La indicación de radioterapia fue por un cáncer cérvico uterino en 9 casos, de próstata en 7 casos y endometrio en 4. La dosis de radiación externa recibida varió entre los 5.600 y 7.000 cGy. Las siete pacientes tratadas por cáncer de cuello uterino recibieron además braquiterapia. Las dosis más altas de radioterapia fueron recibidas por los varones portadores de cáncer de próstata.

El tiempo transcurrido entre la finalización del tratamiento radiante y el inicio de los síntomas fue en promedio de 12 meses, con márgenes entre 8 y 36 meses. No se encontraron diferencias entre la aparición de los síntomas y el motivo de indicación de la radioterapia.

En todos los pacientes el motivo de consulta fue sangrado rectal, siendo catalogado como grave en 15 pacientes quienes presentaban anemia crónica y requerían trasfusiones a repetición. En tres pacientes fue catalogado como moderado ya que pese a presentar sangrado diario y abundante, según la evaluación subjetiva, no presentaba anemia ni requería transfusiones. Los dos pacientes restantes fueron sometidos al tratamiento por presentar patología agregada que requería tratamiento anticoagulante a permanencia.

En cinco pacientes el tratamiento inicial fue en pabellón. El promedio de sesiones necesarias para controlar el sangrado fue de 2, con márgenes de 1 y 3. En tres pacientes se realiza una aplicación con lo que el sangrado se detiene. En 12 pacientes fue necesario realizar una segunda aplicación para lograr la mejoría y en 4 pacientes se efectuaron 3 procedimientos. Estos tres pacientes fueron tratados en pabellón.

Diecinueve pacientes mejoran después del tratamiento. En aquellos que requería transfusiones, se consideró exitoso el tratamiento cuando estas ya no fueron necesarias. En los pacientes que presentaban sangrado sin anemia se consideró el éxito con la desaparición del sangrado rectal. Tabla 1.

Una paciente tratada por un cáncer de endometrio

Tabla 1. Resultados del tratamiento

\begin{tabular}{ccc}
\hline $\begin{array}{l}\text { n de aplicaciones para } \\
\text { controlar sangrado }\end{array}$ & $\mathbf{n}$ & $\mathbf{\%}$ \\
\hline 1 & $4^{*}$ & 20 \\
2 & 12 & 60 \\
3 & 4 & 20 \\
\hline
\end{tabular}

*Un fracaso por perforación en territorio neoplásico. continuó sangrado después del primer procedimiento y luego presentó una perforación de sigmoides en terreno neoplásico, no demostrado en el pre operatorio, a las 48 horas del tratamiento, no se intentó una segunda sesión, por lo que se considera un fracaso del procedimiento. Además un paciente presentó una proctitis intensa con pujo y tenesmo rectal además de pérdida de mucosidades, la que cedió con el tratamiento sintomático en 15 días, con lo que la morbilidad del procedimiento alcanza a un $10 \%$.

El seguimiento promedio es de 36 meses con extremos de 4 y 72 . Los diecinueve pacientes que completaron el tratamiento permanecen asintomáticos, sin sangrado rectal y sin necesitar transfusiones, por lo que el éxito acumulado del tratamiento es de un $95 \%$.

\section{Discusión}

El sangrado rectal después de recibir un tratamiento radiante es una complicación frecuente ${ }^{2}$. Existe una serie de alternativas para evitar el daño rectal por la radioterapia pélvica, pero pese a su utilización la incidencia de esta complicación puede alcanzar a cifras de un $20 \%^{9-11}$.

En su etapa crónica el síntoma más frecuente es el sangrado rectal. Su valor clínico es motivo de discusión, pero existe evidencia que la evaluación endoscópica del recto y del colon es fundamental para certificar el diagnóstico y descartar otra patología colónica ${ }^{12}$. Por este motivo creemos que se encuentra plenamente justificada nuestra conducta de estudiar a todos los pacientes con una endoscopía flexible.

$\mathrm{Al}$ evaluar la gravedad del daño por radioterapia en estos pacientes resulta de escasa utilidad la clasificación propuesta por Sherman ${ }^{13}$, ya que, al excluir los pacientes con úlceras, estenosis y fístulas, todos los pacientes tratados con esta técnica se encuentran en el grado I. De esta forma resulta más útil una evaluación del sangrado en cuanto a frecuencia, cuantía y repercusión en el hematocrito ${ }^{1}$. Otras formas de clasificar, en las que se incluye un puntaje al sangrado y a los hallazgos endoscópicos, nos parecen más difíciles de utilizar por su mayor complejidad ${ }^{14}$.

Las alternativas de tratamiento de esta condición incluyen terapias medicamentosas como los derivados salicílicos y corticoides ${ }^{5}$, enemas de corticoides, ácidos grasos de cadena corta ${ }^{11}$ o sucralfato ${ }^{15} \mathrm{y}$ la utilización de láser de argón ${ }^{16-18}$. Las alternativas medicamentosas orales y a través de enemas no ha demostrado ser de mayor utilidad que el placebo y en cuanto al plasma de argón, su utilidad está demostrada por distintas series no aleatorias, aunque los pacientes tratados, al parecer sangran menos que 
los tratados con formalina, dado que la proporción de pacientes que requieren transfusión es menor ${ }^{14}$. Además debe considerarse el costo, que sin duda es mayor para el láser.

Desde la primera descripción de esta técnica por parte de Rubinstein ${ }^{8}$, han sido numerosos los reportes acerca de su utilidad ${ }^{4,19-21}$. Las tasas de éxito en el control del sangrado rectal reportadas por la literatura están entre el 87 y un 100\% de los casos ${ }^{14}$, lo que se encuentra en concordancia con los datos encontrados por nosotros.

En cuanto a la seguridad de la técnica, desde el punto de vista experimental se ha demostrado, en modelos caninos, que la administración intra rectal, en alícuotas de bajo volumen y por períodos cortos de tiempo, es una técnica segura ${ }^{22}$. Los niveles plasmáticos de formol, pese a elevarse en forma transitoria, no alcanzan niveles tóxicos y retornan a la normalidad en un plazo de tres horas. No se encuentran cambios en la distensibilidad rectal, ni en el contenido de colágeno de la pared del recto. La proctitis inducida por formalina en este modelo cura completamente en un plazo de dos semanas.

Por la alta tasa de curación y escasas complicaciones de la utilización de formalina al 4\% creemos que no se justifica utilizar concentraciones mayores, como han propuesto algunos autores ${ }^{1}$.

$\mathrm{Al}$ analizar la morbilidad de esta técnica también se confirma su seguridad. Las tasas de complicaciones llegan hasta un 25\%, destacando la proctitis aguda sintomática ${ }^{1}$. Otras complicaciones descritas son la estenosis, las úlceras y perforación rectal, aunque estas pueden ser atribuidas a la evolución natural de la enfermedad. En nuestra serie la morbilidad llegó a un 15\%. En esta está incluido un caso de perforación de sigmoides en terreno neoplásico. La recidiva no fue demostrada en el estudio previo. Esta complicación puede no tener relación alguna con la aplicación de formalina, más aún si se considera que esta paciente fue tratada en pabellón y en posición de litotomía, con lo que la probabilidad del avance retrógrado de la formalina se minimiza. Además la biopsia de la pieza operatoria no mostró elementos agudos en la mucosa que pudieran ser atribuidos a una sigmoiditis por formalina. El segundo caso es una proctitis aguda que respondió al tratamiento con dieta y analgésicos.

\section{Conclusión}

Basados en los hallazgos de esta serie prospectiva no aleatoria, creemos que la aplicación de formalina tópica al 4\% es una técnica efectiva y segura para el tratamiento del sangrado rectal de la proctitis inducida por radioterapia.

\section{Referencias}

1. Haas EM, Bailey HR, Farragher I. Application of 10 percent formalin for the treatment of radiation-induced hemorrhagic proctitis Dis Colon Rectum 2006;50: 213-7.

2. Johnston MJ, Robertson GM, Frizelle FA. Management of late complications of pelvic radiation in the rectum and anus Dis Colon Rectum 2003;46:247-59.

3. Corman ML, Vascular diseases. In Corman ML. ed. Colon and rectal surgery. $4^{\text {th }}$ ed. Philadelphia: JB lippincot, 1989;1060-4.

4. Seow-Choen F, Goh H-S, Eu K-W, Tay S-K. A simple and effective treatment for hemorrhagic radiation proctitis using formalin. Dis Colon Rectum 1993;36:135-8.

5. Goldstein F, Khoury J, Thorton JJ. Treatment of chronic radiation enteritis and colitis with salicylazopyridine and systemic corticosteroids. Am J Gastroenterol. 1976;65:202-8.

6. Smith S, Wallner K, Dominitz JA, Han B, True L, Sutlief S, et al. Argon plasma coagulation for rectal bleeding after prostate brachytherapy. In J Radiat Oncol Biol Phys. 2001;51:636-42.

7. Brown RB. A method of management of inoperable carcinoma of the bladder. Med J Aust. 1969;1:24-4.

8. Rubinstein E, Ibsen $\mathrm{T}$, Rasmussen RB, Reimer E, Sorenson BL. Formalin treatment of radiation induced hemorrhagic proctitis. Am J Gastroenterol. 1986;81:445.

9. Cho KH, Lee C, Levitt SH. Proctitis after conventional external radiation therapy for prostate cancer: Importance of minimizing posterior rectal dose. Radiology 1995;195:699-703.

10. Bem J, Bem S, Singh A. Use of hyperbaric oxygen chamber in the management of radiation-related complications of the anorectal region. Report of two cases and review of the literature. Dis Colon Rectum 2000;43:1435-8.

11. Talley NA, Chen F, King D, Jones M, Talley NJ. Shortchain fatty acids in the treatment of radiation proctitis. A randomized, double-blind, placebo controller, crossover pilot trial. Dis Colon Rectum 1997;40:1046-50.

12. Williams HTR, Vlavianos P, Blake P, Tait D, Andreyev HJN. The significance of rectal bleeding after radiotherapy. Aliment Pharmacol Ther. 2005;21:1085-90.

13. Sherman LF. A reevaluation of the factorials proctitis problem. Am J Surg. 1954;88:773-9.

14. Zinicola R, Rutter M, Falasco G, Broker J, Cennamo V. Haemorraghic radiation proctitis: Endoscopic severity may be useful to guide therapy. Int J Colorrectal Dis. 2003;18:439-44.

15. Hovdenak N, Sorbye H, Dahl H. Sucralfate does not ameliorate acute radiation proctitis: randomized study and meta-analysis. Clin Oncol. (R Coll Radiol) 2005;17:485-91.

16. Silva RA, Correia AJ, Dias LM, Vianna HL, Vianna 
A. BARRERA E. y cols.

RL. Argon plasma coagulation therapy for hemorrhagic radiation proctosigmoiditis. Gastrointest Endosc. 1999;50:221-4.

17. Fantin AC, Binek J, Suter WR, Mayenberger C. Argon beam coagulation for treatment of symptomatic radiation-induced proctitis. Gastrointest Endosc. 1999;49:515-8.

18. Tam W, Moore J, Schoeman M. Treatment of radiation proctitis with argon plasma coagulation. Endoscopy 2000;32:667-72.

19. Biswal BM, Lal P, Rath GK, Shukla NK, Mohanti BK. Intrarectal formalin, an effective treatment for grade III hemorrhagic radiation proctitis. Radiother Oncol.
1995;35:212-5.

20. Saclarides TJ, King DG, Franklin JL, Doolas A. Formalin instillation for refractory radiation-induced hemorrhagic proctitis: report of 16 cases. Dis Colon Rectum 1996;39:196-9.

21. Yeggapan M, Ho YH, Nyam D, Leong A, Eu KW. The surgical management of colorrectal complications from irradiation for carcinoma of the cervix. Ann Acad Med Singapore 1998;27:627-30.

22. Myers JA, Hollinger EF, Mall JW, Jakate SM, Doolas A, Saclarides TJ. Mechanical, histological, and biochemical effects of canine rectal formalin instillation. Dis Colon Rectum 1998;41:153-8. 